\title{
Surface microgeometry treated with a precast textured grinding wheel
}

\author{
Vladimir Gusev* \\ Vladimir State University named after Alexander and Nikolay Stoletovs, Russian Federation
}

\begin{abstract}
The article considers the formation of the geometry of internal cylindrical surfaces when grinding with a precast textured wheel, which is under the influence of the unbalances main vector and a variable cutting force caused by the discreteness of the cutting surface (texture). Under the influence of these factors, each point of the axis of the textured tool makes vibrations in the transverse plane in the form of a wavelike sinusoid consisting of two sinusoids. The Space-time process of forming the processed surface is mathematically described. It is in applying wavelike sinusoids to the workpiece, taking into account their phase shift at each revolution of the workpiece. To ensure minimal geometric errors at the maximum possible productivity of the grinding process, phase shifts $\varphi_{\mathrm{f}}=$ $(0.07-0.12) \pi$ and $\varphi_{\mathrm{f}}=(0.88-0.93) \pi$ are recommended. The results of the study are recommended for use in the production of high-precision details, primarily from materials that are prone to thermal damage to the surface layer under the influence of high temperature in the grinding zone.
\end{abstract}

\section{Introduction}

The processes of grinding metals and alloys are characterized by high geometric accuracy of the processed surfaces, low roughness and undulation [1-5], as a result of which a large amount of grinding operations is widely used in the industry. The main disadvantage of grinding is the high thermal stress in the cutting zone [6], which causes thermal damage to parts, reducing their operational reliability. Leveling this disadvantage is achieved by reducing the grinding modes, using a tool with two grinding wheels (coarse-grained and finegrained) [7], using soft, highly porous [8], single-component [9], discrete wheels [1-3, 1012], high-precision balancing, effective methods and means of cooling the ground workpieces [13, 14], etc. The most actively researched the textured grinding wheels [15-17], which are of great importance for both academic science and industry [15].

Precast textured grinding wheels reduce the local temperature of the processed surface of the workpiece up to 3 times [1], which is due to a combination of discontinuity of the cutting surface and the intensification of heat removal by accelerating the coolant to approximately the cutting speed and directing it to the local areas of contact between the tool and the workpiece. In [18], the stability of the grinding process is analyzed taking into account its dynamic characteristics, in [19] - the dynamics of grinding with discrete wheels, in [20] - the

*Corresponding author:prof_gusev@mail.ru 
formation of surfaces during abrasive processing with a standard tool. At the same time, the study of the treated surface formation in the process of grinding by perspective precast textured wheels was not given due attention, which does not allow us to assign cutting modes that provide the required microgeometry of surfaces at the maximum possible productivity of the processing process.

The purpose of this study is to reveal the mechanism of geometry formation and establish a multi-factor experimental model of surface roughness treated with a precast textured tool, on the basis of which it is possible to assign a grinding modes that provide the required values of microgeometry at the maximum possible productivity of the processing process.

\section{Methods and Materials}

When analyzing the mechanism of surface geometry formation, scientific methods of vibration theory in technological systems, material grinding, spatio-temporal surface shaping were used. Multifactorial experimental studies were made on internal grinding machines 3A227B and SWIGLE-250. Were processed details of serial and mass production: rings of bearings 114, 7214 from steel SHH15, HRC 62-65; cones loading mechanism of blast furnaces covered by hard alloys PL-AN 111, 121.

Characteristics of the abrasive segments of the precast textured grinding wheel 25AF90KV5. Akvol-10M emulsion was used as a coolant liquid. The influence of design factors of the precast textured wheel and elements of the grinding mode on the roughness of the surface, which was measured on the device "Surtronic" (Denmark), was studied.

\section{Results}

A system of differential equations of motion of a precast textured grinding wheel was compiled and solved. as a result, it was found that under the action of the main vector of unbalances $D_{\text {st }}$, the center of the wheel fixed on the machine spindle moves in space according to the sinusoidal law:

$$
Y_{1}=A_{1} \sin (\omega \tau+\varphi)
$$

where $A_{1}=Q / J_{r}=\left(m \omega^{2} \rho\right) / J_{r}-$ amplitude oscillations of the center of the wheel under the action of the main vector of unbalances $D_{s t}=m \rho ; Q$ - unbalanced centrifugal force caused by the action of the vector $D_{\text {st }} ; m, \rho$ - respectively, the unbalanced mass and the radius of its gravity centre; $\omega, \tau$ - respectively the angular velocity and the current time of grinding; $\mathrm{J}_{\mathrm{r}}$ - rigidity of spindle assembly of a grinding machine tool in a direction perpendicular to the processed surface; $\varphi$ - angle of the main vector of unbalances $D_{\text {st }}$.

Under the influence of unbalances and discontinuity of the cutting surface of a prefabricated textured wheel, the movement of its center is described by the equation:

$$
Y_{0}=A_{1} \sin (\omega \tau+\varphi)+A_{2} \sin (\omega \tau+\varphi) n_{s}
$$

where $n_{s}-$ is the number of abrasive segments in the precast textured wheel.

The geometric interpretation (2) is represented by a wavelike sinusoid, which consists of a low-frequency and a high-frequency sinusoids. Wavelike sinusoid in the process of discrete grinding is applied to the surface to be processed during each revolution of the workpiece. Let's call a wavelike sinusoid deposited on the first revolution of the workpiece, zero, then the equation of the first wavelike sinusoid deposited during the second revolution of the workpiece, it will take the form:

$$
Y_{1}=A_{1} \sin \left(\varphi+x+\varphi_{1}\right)+A_{2} \sin \left(\varphi+x+\varphi_{1}\right) n_{s}
$$


where $\mathrm{x}=\omega \tau-$ is the angle of rotation of the main vector of unbalances $\mathrm{D}_{\text {st }}$ during grinding; $\varphi_{1}-$ is the phase shift of the zero and first wavelike sinusoids on the first and second revolutions of the workpiece.

The grinding mode, in which the $2 \mathrm{nd}, 3 \mathrm{rd}$, and so on sinusoids are displaced by $\pi$ radians, is characterized by a high geometric error $\Delta_{1}$, which is a disadvantage, so this mode should be excluded. The grinding mode, in which the 2 nd and subsequent sinusoids are shifted in phase by an angle of $0.33(3) \pi$, is characterized by a slow correction of the error $\Delta_{1}$ and a decrease in its value after each subsequent revolution of the workpiece (Fig. 1).

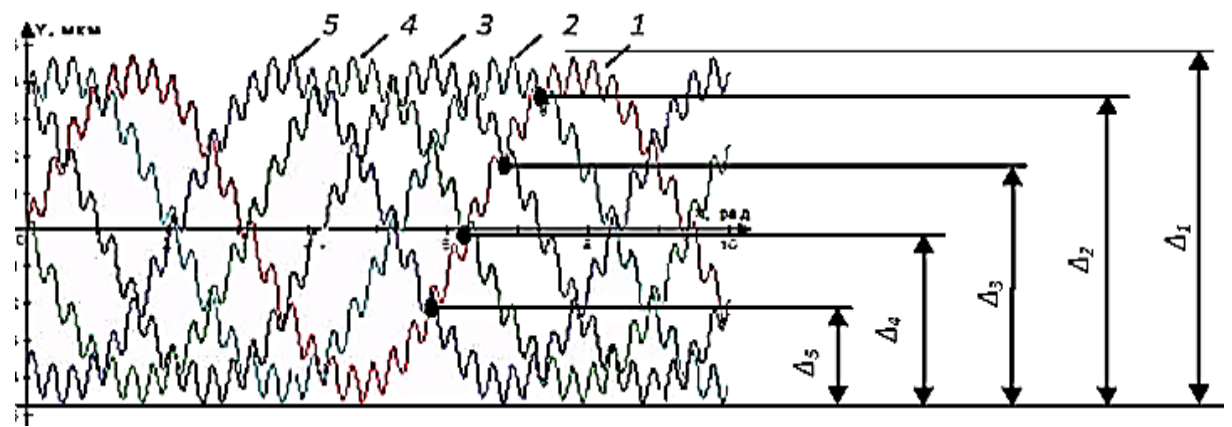

Fig. 1. Surface formation with a phase shift of adjacent sinusoids equal to $0.33(3) \pi$.

Profile of the processed surface when shifting adjacent sinusoids $0.33(3) \pi$ radian is described by the equations:

$$
\begin{aligned}
& Y=4.2 \sin x+0.5 \sin (22 x) \text { for } 0 \leq x \leq(\pi / 3) k, \\
& Y=4.2 \sin (x+\pi / 3)+0.5 \sin (22 x) \text { for }(\pi / 3) k \leq x \leq(2 \pi / 3) k, \\
& Y=4.2 \sin (x+2 \pi / 3)+0.5 \sin (22 x) \text { for }(2 \pi / 3) k \leq x \leq \pi k, \\
& Y=4.2 \sin (x+\pi)+0.5 \sin (22 x) \text { for } \pi k \leq x \leq(4 \pi / 3) k \\
& Y=4.2 \sin (x+4 \pi / 3)+0.5 \sin (22 x) \text { for }(4 \pi / 3) k \leq x \leq(5 \pi / 3) k, \\
& Y=4.2 \sin (x+5 \pi / 3)+0.5 \sin (22 x) \text { for }(5 \pi / 3) k \leq x \leq(2 \pi) k .
\end{aligned}
$$

If there is no phase shift, i.e. at $\varphi_{\mathrm{f}}=0$, the final error of the treated surface takes the maximum possible value of 17.2 micrometer (Fig. 2, line 1), and at $\varphi_{\mathrm{f}}=\pi$ the final error is less than the maximum (line 2).

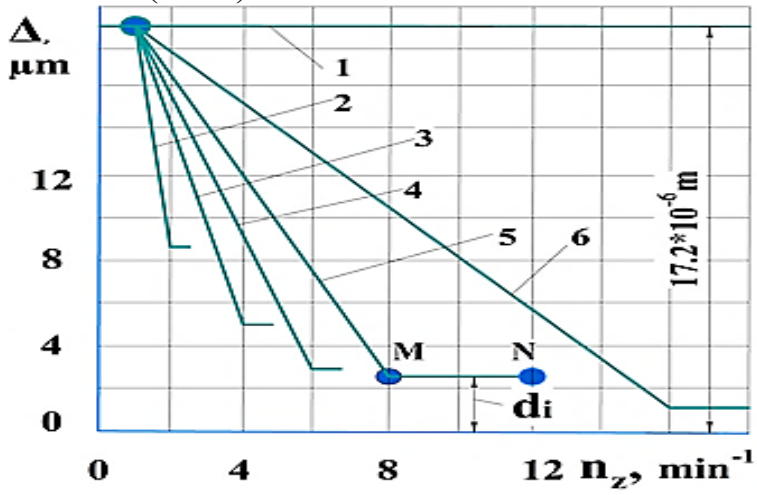

Fig. 2. Reduction of the geometric error of the processed surface with an increase in the number of the workpiece revolutions in the cutting zone. 
Lines 3-6 characterize the decreasing of the maximum error $\Delta_{1}$, respectively, when shifting $\varphi_{\mathrm{f}}=\pi / 2, \varphi_{\mathrm{f}}=\pi / 3, \varphi_{\mathrm{f}}=\pi / 4$, and $\varphi_{\mathrm{f}}=\pi / 8$. The smaller the number of forming revolutions of the workpiece, the faster the surface formation ends and Vice versa. For lines $2-6$, the final errors due to the main vector of unbalances $D_{\text {st }}$ and the discretization of the cutting surface of the tool are: $\Delta_{2}=8.6 ; \Delta_{3}=5.1 ; \Delta_{4}=3.3 ; \Delta_{5}=2.4$, and $\Delta_{6}=1.1 \mu \mathrm{m}$. After the workpiece performs forming revolutions, the error does not decrease after an arbitrarily large number of additional revolutions.

Thus, when the phase shift between adjacent wavelike sinusoids decreases, the geometric error of the processed surface decreases, but the processing time of the workpiece increases, which leads to a decrease in the productivity of the process. The information contained in Fig. 2 is very important, because it, first, contains data on the geometric accuracy of the surface, and secondly, from Fig. 2, you can determine the number of revolutions $\mathrm{n}_{\mathrm{z}}$ of the workpiece, which must be performed to ensure the geometric accuracy $\Delta_{\mathrm{d}}$, set by the drawing of the part.

Using the known value $\Delta_{\mathrm{d}}$, we select a line whose distance $\mathrm{d}_{\mathrm{i}}$ of the horizontal segment MN from the abscissus axis is less than or equal to the value $\Delta_{\mathrm{d}}$. The number of forming revolutions of the workpiece nzm is equal to the value of the abscissa of point M. For example, for a line 5, abscissa of the point $\mathrm{M}$ is 8 , so for the grinding mode with a shift of wavelike sinusoids $\varphi_{\mathrm{f}}=\pi / 8$, the value $\mathrm{n}_{\mathrm{zm}}=8$ revolutions.

The known number of forming revolutions $\mathrm{n}_{\mathrm{zm}}$ determines the minimum time for grinding the workpiece to ensure the required geometric accuracy of the surface. The phase shift $0,05 \pi$ $\leq \varphi_{\mathrm{f}} \leq 0.10 \pi$ in comparison with the shifts $\varphi_{\mathrm{f}}=\pi / 2, \varphi_{\mathrm{f}}=\pi / 3, \varphi_{\mathrm{f}}=\pi / 4, \varphi_{\mathrm{f}}=\pi / 8$ provides the smallest geometric error of the surface and the longest grinding time of the workpiece. The results of experimental studies conducted in accordance with the above methodology showed a satisfactory convergence with the data of the theoretical analysis of the process of forming the treated surface (the differences were no more than 12\%).

For 25AF90KV5 abrasive segments, the multi-factor experimental model of the arithmetic mean deviation of the profile $\mathrm{Ra}$ of the treated surface has the form:

$$
\mathrm{Ra}=-1.565+0.762 * \mathrm{~S}_{\mathrm{r}}+1.28 * 10^{-3} \mathrm{n}_{\mathrm{z}}+0.03 * \mathrm{D},
$$

where $\mathrm{S}_{\mathrm{r}}, \mathrm{n}_{\mathrm{z}}$ - are the radial feed and rotation frequency of the workpiece, respectively; $\mathrm{D}$ - is the diameter of the cutting surface of the textured wheel.

The graphical interpretation of the model (5) is shown in Fig. 3, which shows that the dominant influence on microgeometry affacts the radial feed $S_{r}$ of the workpiece, then the degree of influence on the parameter Ra is followed by the rotation frequency $n_{z}$.
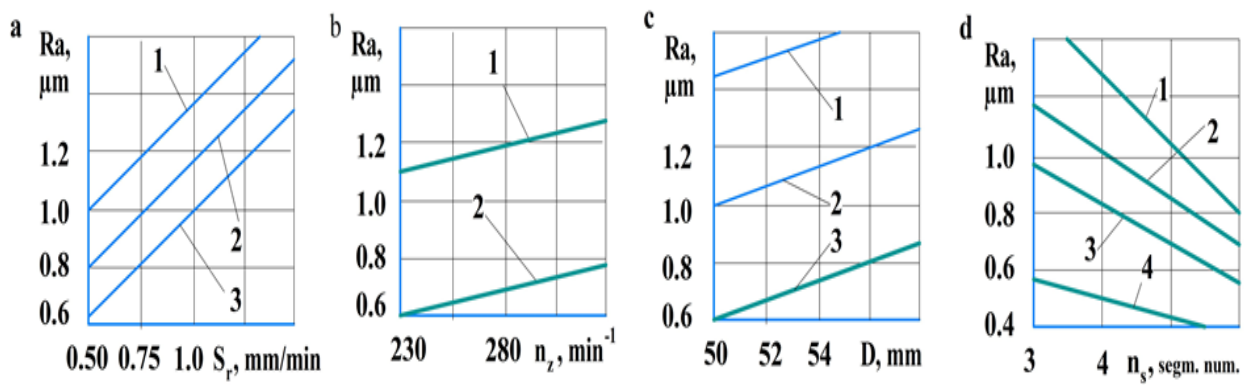

Fig. 3. Influence on the parameter $R a$ of the radial feed $S_{r}$ (a), rotation frequency $n_{z}$ of workpiece (b), the diameter D of the cutting surface (c), and segments number in the textured wheel (d). 
As the factors $\mathrm{S}_{\mathrm{r}}, \mathrm{n}_{\mathrm{z}}$, and $\mathrm{D}$ increase, the surface roughness increases in accordance with the regression equations:

Fig. 3a. $1 . R a=0.6+0.76 * S_{r} ; 2 \cdot R a=0.4+0.76 * S_{r} ; 3 . R a=0.23+0.76 * S_{r}$;

Fig. 3b. $1 . R a=0.82+1.28 * n_{z} ; 2 . R a=0.32+1.28 * n_{z}$;

Fig. 3c. $1 . R a=0.03 * D ; 2 . R a=-0.45+0.03 * D ; 3 . R a=-0.89+0.03 * D$.

A significant influence on the microgeometry of the surface has the number of $n_{s}$ abrasive segments in the textured wheel, when it increases the roughness decreases (Fig. 3, d). Straight lines $1-4$ correspond to the grinding of workpieces with a radial feed $\mathrm{S}_{\mathrm{r}}=2.5 \cdot 10^{\wedge}(-2)$; $2.0 \cdot 10^{\wedge}(-2) ; 1.3(3) \cdot 10^{\wedge}(-2) ; 6.6(6) \cdot 10^{\wedge}(-3) \mathrm{mm} / \mathrm{s}$, respectively. The minimum values of the parameter Ra are obtained when the phase shift of neighboring wavelike sinusoids $\varphi_{\mathrm{f}}=(0.07$ $-0.12) \pi$ and $\varphi_{\mathrm{f}}=(0.88-0.93) \pi$.

\section{Discussion}

As a result of the analysis of the spatio-temporal process of forming a surface processed with a precast textured wheel, it is established that geometric errors (irregularities) occur during grinding, the value of which depends on the cutting mode and design factors that characterize the tool. Each grinding mode and the design of the tool determine the value of the phase shift of wavelike sinusoids and the number of forming revolutions of the workpiece in the cutting zone. The forming revolution - is a revolution of the workpiece during which the geometric error of the surface is reduced. The closer the shift value phases of adjacent wavelike sinusoids is to zero or one, the lower the final geometric error and processing performance. This is due to the fact that at phase shifts $\varphi_{\mathrm{f}}=(0.07-0.12) \pi, \varphi_{\mathrm{f}}=(0.88-0.93) \pi$, material removal at each forming revolution of the workpiece occurs in small volumes, which is typical for the finishing mode of grinding, and at $\varphi_{\mathrm{f}}=\pi$ - much larger volumes, typical for pre-processing.

An increase in the number of abrasive segments in a textured wheel leads to a decrease in the parameter Ra (Fig. 3, d), which is explained by an increase in the frequency of single cuttings applied by segments to the workpiece. Regression equations (5)-(8) represent an experimental multi-factor model of the parameter $\mathrm{Ra}$ and allow controlling the microgeometry of the surface as a function of the cutting mode elements and design factors of a prefabricated textured tool that provides a centrifugal supply of coolant liquid to the cutting zone.

The specifics of the experimental research results are not only the established multi-factor model of the parameter Ra, but also the increase in roughness with increasing diameter of textured tool. For grinding processes with a standard wheel, the parameter Ra decreases as the cutting surface diameter increases, which is explained by an increase in the number of cutting abrasive grains and a decrease in the volume of material removed by a single grain. When processing with a precast textured wheel with a centrifugal coolant supply to the cutting zone through a rapidly rotating tool, with an increase in the diameter $\mathrm{D}$, the coolant dispersion increases, its efficiency decreases, which affects the increase in roughness.

\section{Conclusions}

1. The Formation of the processed surface during grinding with a precast textured wheel, was not given due attention, which does not allow you to assign cutting modes that provide the required microgeometry of the surfaces at the maximum possible performance of the processing process. 
2. As a result of analysis of the spatio-temporal process of forming the surface by precast textured wheel establiched a significant influence on geometric error of the phase shift between adjacent wavelike sinusoids characterizing the vibration textured wheel under the action of the main vector of unbalances and discretization of cutting surface. As a result of the disclosure of the mechanism for forming the treated surface, it was found that in order to ensure minimal geometric errors at the maximum possible performance of the grinding process, phase shifts $\varphi_{\mathrm{f}}=(0.07-0.12) \pi$ and $\varphi_{\mathrm{f}}=(0.88-0.93) \pi$ are recommended,

3 . The results of the multi-factor experiment showed satisfactory convergence with the data of the theoretical analysis of the process of forming the treated surface. A multi-factor experimental model of the arithmetic mean deviation of the profile $\mathrm{Ra}$ of the processed surface in the function of independent factors characterizing the cutting mode and the tool design has been established, which allows controlling the microgeometry of the treated surface. The research results are recommended for use in the production of high-precision machine parts, including prone to thermal damage to the surface layer under the influence of high temperature in the grinding zone.

\section{References}

1. V. Gusev, V. Morozov, RU, St. un-ty, Vladimir (2007)

2. V. Gusev, A. Morozov, RU, Yoshkar-Ola Pub. House (2012)

3. A. Morozov, V. Gusev, Pen Publ. House, Moscow (2016)

4. I. Koltunov, Iu. Stepanov, A. Tarapanov, Mech. Eng.-1, Moscow (2007)

5. Z. Kremen, Yuryev V, Pub. house of polytech. un-ty, St. Petersburg (2007)

6. Z. Kremen, V. Iuryev, Pub. house of tech. un-ty, St. Petersburg (2013)

7. Gusev V.G., Morozov A.V., Grinding method RU Patent 2,606,143, Sept. 2016

8. V. Starkov, Moscow. Mech. Eng., Moscow (2007)

9. Ju. Poljanchikov, Dissertation, RU, Saratov Un-ty (2002)

10. V.G. Gusev, A.V. Morozov RU J Sci. Int. Tech. in Mech. Eng. 9(73): 20-27 (2017)

11. V.G. Gusev, A.V. Morozov, P.S. Shvagirev, J RU Eng. Res. 29(9): 940-943 (2009)

12. A.V. Morozov, RU J. Sci. Eng. industry bul. 3:59-64 (2016)

13. L. Khudobin, A. Babichev, E. Bulyzhev, Mech. Eng., Moscow (2006)

14. A. M. Dolgano, All-RU sci. tech. pr. conf. Novosibirsk (2006)

15. H. Li, D. Axinte, A review. Int. J of Mach. Tools and Manufacture Vol. 109: 8-35. doi: http://dx.doi.org/10.1016/j.ijmachtools.2016.07.001

16. A. Daneshi, B. Azarhoushang, Mat. Sci. Forum, Vol. 874:101-108 Trans Tech Publ., Switzerland (2016)

17. D. Blurtsyan, Mat. Sci. Forum Vol. 874:85-91, Trans Tech Publ., Switzerland (2016)

18. Iu. Zubarev, A. Priemyshev, Publ. house of polytech. un-ty, St. Petersburg (2016)

19. V.G. Gusev, A.V. Morozov, P.S. Shvagirev, J Sci. Rus. Eng. Res. 29(8):835-837 (2009)

20. Iu. Novoselov, RU Nat. tech. un-ty, Sevastopol (2012) 